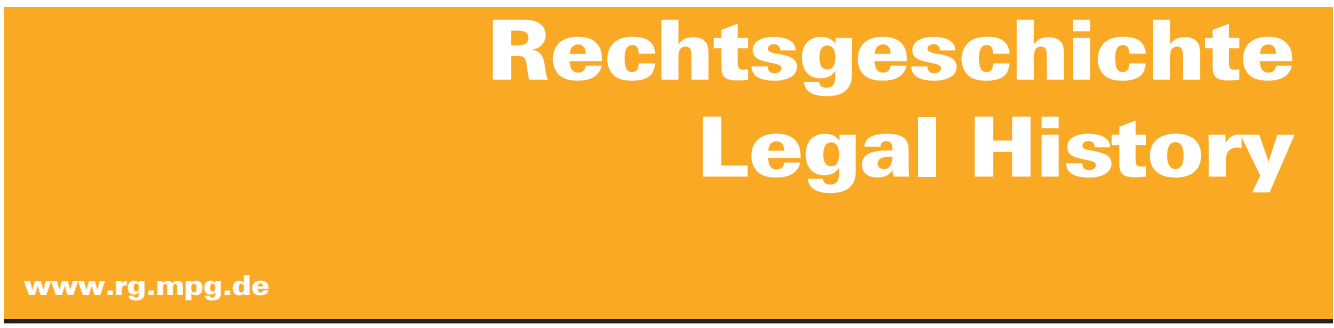

http://www.rg-rechtsgeschichte.de/rg23

$\operatorname{Rg} 232015$

$256-259$

Zitiervorschlag: Rechtsgeschichte - Legal History Rg 23 (2015)

http://dx.doi.org/10.12946/rg23/256-259

\title{
Wolfgang Ernst
}

\section{Zur Epistemologie rechtsgeschichtlicher Forschung}




\section{Wolfgang Ernst Zur Epistemologie rechtsgeschichtlicher Forschung}

[1] Da es der rechtsgeschichtlichen Forschung um das Recht der Vergangenheit geht, ist sie in ihrer Epistemologie von der Existenzform des Phänomens Recht abhängig. Alles Recht ist gedanklicher Natur. Es handelt sich um die Überzeugung, dass für das organisierte menschliche Zusammenleben gewisse Regeln verpflichtender Art zu beachten sind, deren Verletzung mit einer Sanktion geahndet werden wird/soll. Wird Recht in einem objektiven Sinne (»Rechtsordnung«) angesprochen, handelt es sich um ein Konstrukt. Überzeugungen sind als solche individuell. Eine »Kollektivüberzeugung « hat keine reale Existenz. Überindividuell handelt es sich um ein Kommunikationsgeschehen, in dem eine teilweise oder weitgehende Übereinstimmung darüber besteht oder hergestellt wird, was als Recht und was als Unrecht anzusehen ist. In fortgeschrittenen Gesellschaften nehmen an diesem Kommunikationsgeschehen Personen teil, die in verschiedenen Rollen mit Vertragsgestaltung, Streitentscheidung, Normerzeugung und akademischer (intergenerationeller) Normvermittlung befasst sind. Die überindividuelle Existenz von Recht als einem Gedankengebilde, das weithin übereinstimmend für zutreffend gehalten wird, ist ein Tatbestand, der angemessen nur wissenssoziologisch erfasst werden kann.

[2] Das Recht als Gedankengebilde - oder genauer als ein wissenssoziologisches Gesamtgeschehen gedanklich zusammenwirkender Personen ist in vielfältiger Weise in die physikalische Welt eingebunden. Jede Hervorbringung von Gedankeninhalten ist von Geschehnissen in der physikalischen Welt abhängig und begleitet: Auf eine Urkunde wird ein Siegel gesetzt, ein Urteil wird viva voce verkündet, die Gefängnistür schließt sich hinter dem Strafgefangenen. Dergleichen Vorgänge sind nicht das Recht, sondern es sind Hand- lungen, für welche Überzeugungen davon, was rechtens ist, (mit-)bestimmend geworden sind. Die in der physikalischen Welt greifbaren Erscheinungen, in denen sich Rechtsüberzeugungen ausgedrückt haben, dürfen nicht mit dem Recht als einem Gedankengebilde identifiziert werden: Indem man die Vorgänge in einem Gerichtsgebäude oder in einer Anwaltskanzlei filmt, kann man nicht das erfassen, was wir als »Recht « ansprechen. Ebenso ist Vornahme eines juristischen Schriftstücks aus der näheren oder ferneren Vergangenheit nicht schon Wahrnehmung von Recht, sondern es erschließt sich etwas vom Recht, wenn im Vorgang des Verstehens dem Schriftstück juristische Aussagen - also Gedankeninhalte - entnommen werden. Recht ist und war zwar von "Medialität» begleitet, aber das Recht als Gedankengebilde darf mit seiner Medialität nicht verwechselt oder gleichgesetzt werden.

[3] Gegenstand der rechtsgeschichtlichen Forschung ist vergangenes Handeln, das durch eine zeitgenössische Vorstellung von Recht und Unrecht bestimmt oder mitbestimmt worden ist. Zum Gegenstand rechtsgeschichtlicher Forschung wird ausgewähltes Geschehen der Vergangenheit gerade wegen der handlungsleitenden Rechtsvorstellung; diese ist es, die das spezifisch rechtsgeschichtliche Interesse auslöst. ${ }^{\mathbf{1}}$ Insofern gilt: Alle Rechtsgeschichte ist Geistesgeschichte. Daher ist "Verstehen« ein unverzichtbares Zentralmoment auch der rechtsgeschichtlichen Forschung. Und wo es um einen spezifisch rechtlichen Gedankeninhalt geht, ist auch ein juristisches Verstehen gefordert.

[4] Die Isolierung von Rechtsgeschichte (wie die anderer Spezialgeschichten auch) reißt ursprünglich Zusammenhängendes auseinander. Dergleichen Isolierungseffekte sind unvermeid-

1 Von der rechtsgeschichtlichen Forschung kann auch solches Handeln in den Blick genommen werden, das bestimmte Rechtsvorstellungen lediglich zum »Hintergrund « hat, etwa der Abschluss eines Vertrages, bei dem die Beteiligten wissen, wie sie sich der Gerichtsfestigkeit des Vereinbarten versichern. 
lich, weil das vergangene Geschehenskontinuum in seiner Totalität nicht rekonstruierbar ist (wie aber schon der Zeitgenosse sich das Gesamtgeschehen nicht in seiner Totalität verfügbar machen kann, sondern nur ordnend und auswählend also in einzelnen Hinsichten und unter Abstrahierung von nicht interessierenden Details).

[5] Gegenstand der Geschichtsforschung ist vergangenes Geschehen nur insoweit, wie es durch informationsträchtige Überreste (Quellen) rekonstruiert werden kann. Grundlegend für die Epistemologie rechtsgeschichtlicher Forschung ist der Hiatus zwischen den rechtshistorisch aufschlussreichen Quellen als dem gleichsam empirischen Bezugspunkt unserer Erkenntnissuche und dem historischen Rechtsgeschehen, das sich als vergangener lebendiger Kommunikationsprozess jeder »Beobachtung« entzieht. Eine Gesetzespublikation z. B. in der Gesetz-Sammlung für die Königlichen Preußischen Staaten ergibt selbst hinsichtlich ihres ureigenen Inhalts (»Text«) nicht unmittelbar »das« Recht der Zeit; wohl erschließt sich damit ein fragmentarisches Einzelgeschehen, das im Zeitpunkt seines aktuellen Vorkommnisses auf vielfältige Weise in ein weiteres, von zahlreichen Akteuren getragenes Rechtsgeschehen eingebunden war und aus diesem Verbund seine Bedeutung bezog. Mit dieser Beschränkung auf äußerst fragmentarische Einzeleinblicke muss der Rechtshistoriker sich abfinden. Erkenntnisinteressen hinsichtlich vergangener Rechtsvorstellungen, die sich nicht am Quellenmaterial erschließen lassen, kommen für das wissenschaftliche Arbeiten nicht in Betracht.

[6] Das Wort von einer (gegenwärtigen oder vergangenen) »Rechtswirklichkeit« ist irreführend, indem es suggeriert, es ließe sich ein »wirkliches", in die Lebenswelt eingreifendes Rechtsgeschehen von »bloß « gedanklichen Hervorbringungen abgrenzen, die demnach irgendwie »unwirklich« sein müssten. Es macht epistemologisch keinen Unterschied, ob das von einer Rechtsvorstellung geleitete oder mitbestimmte Handeln (das wir mittels Überresten wieder herstellen können) in der Abfassung einer Prozessschrift oder eines Urteils bestand oder in der Niederschrift eines Vertrages durch einen Notar oder in einer juristischen $\mathrm{Ab}$ handlung oder in der Ausarbeitung einer nie in Kraft gesetzten Gesetzesbestimmung. Der Umstand, dass wir einem vergangenen Geschehen eine »rechtliche« Natur beilegen, ist immer auf der geistigen Ebene angesiedelt. Das freisprechende
Urteil des 17. Jahrhunderts gibt dem vergangenen Rechtsdenken keinen irgendwie geringeren Ausdruck, bloß weil es nicht auch noch durch eine Vollstreckung in die weitere Lebenswirklichkeit eingegriffen hat.

[7] Die - in vielerlei Hinsicht diskussionsbedürftige - Unterscheidung zwischen Rechtspraxis und Rechtsgelehrsamkeit kann man auch in bestimmte Epochen der Vergangenheit hineintragen. In epistemologischer Hinsicht besteht indes kein grundlegender Unterschied in der Erforschung von Produkten vergangener Rechtspraxis und solcher vergangener Rechtsgelehrsamkeit. Keinesfalls ist die vergangene Rechtspraxis »lebenswirklicher« und damit einer geschichtlichen Erforschung näherliegender. Produkte vergangener Literatur, die seinerzeitigen Rechtsvorstellungen Ausdruck gaben (»Theorie«), sind selbst dann valable Gegenstände der rechtsgeschichtlichen Forschung, wenn sie für die »Praxis« gänzlich folgenlos geblieben sein sollten; man denke an unveröffentlichte Buchmanuskripte oder Vorlesungsmitschriften. Im Bereich der romanistischen Forschung wurden in jüngerer Richtung eindrucksvolle Resultate übrigens gerade aufgrund eines Ansatzes gewonnen, der Urkunden mit ihren Vereinbarungen (»Praxis«) und entsprechende Aussagen der juristischen Literatur (»Theorie«) konfrontiert.

[8] Aussagen auf dem Gebiet der rechtsgeschichtlichen Forschung lassen sich nur treffen, wo wir Überreste von in Handlungen ausgedrückten Rechtsvorstellungen finden; die große Zahl der Handlungen, bei denen dies der Fall gewesen ist, die uns aber keine quellenmäßige Spur hinterlassen haben, bleibt außer Betracht. Nicht Gegenstand der Rechtsgeschichte sind hypothetische juristische Gedanken, die nicht nachweislich in einer quellenbelegten Handlung Ausdruck gefunden haben.

[9] Für die heutige Arbeit des Juristen spielt die Vorstellung der Lückenlosigkeit der Rechtsordnung eine gewisse Rolle. Es handelt sich um eine regulative Idee, die ihrerseits ein (diskutables) Element unserer juristischen Gedankenwelt ist. Wenn der Gegenwartsjurist eine bislang unbekannte Rechtsaussage macht, die er - geleitet von der Idee der Lückenlosigkeit der Rechtsordnung - anhand des vorfindlichen Bestandes von Rechtsaussagen so zu gewinnen sucht, dass sich die neue Rechtsaussage in die gedachte Rechtsordnung einfügt und damit an deren Geltungsanspruch teilhaben kann, wird tatsächlich eine neue gedankliche Realität 
erzeugt. Dergleichen ist dem Rechtshistoriker versagt. Er ist kein Teilnehmer am wissenssoziologischen Prozess z. B. des 17. Jahrhunderts. Er kann durch eigene Überlegungen keine vergangenen gedanklichen Realitäten erzeugen und dadurch etwaige Lücken in früheren Rechtszuständen schließen. Dies gilt sowohl, wenn die "Lücke» darin bestehen sollte, dass das entsprechende Thema in der Vergangenheit überhaupt nicht Gegenstand von aktuellen Überlegungen gewesen ist, als auch, wenn unsere fragmentarische Überlieferungslage uns insoweit einfach nichts erkennen lässt. Mit einer Ergänzung der vergangenen Rechtsordnung derart, dass im Wege einer Schlussfolgerung für eine vergangene Rechtsordnung eine Rechtsaussage getroffen wird, für die es einen quellenmäßigen Beleg nicht gibt, wird der Bereich der rechtsgeschichtlichen Forschung verlassen. Ein Religionshistoriker kann ja auch nicht selber als Augur tätig werden.

[10] Die einzelne Quelle kann in aller Regel nicht alleine aus sich selbst heraus verstanden werden. Quervergleiche zwischen verschiedenen Quellen sind unverzichtbar. Inschriftliche Abkürzungen etwa können nur aufgelöst werden, weil die einzelne Inschrift vor dem Hintergrund einer großen Masse vergleichbarer Inschriften verstanden werden kann. Das frühneuzeitliche Urteil zitiert Stellen aus der gelehrten Literatur: Dieses Zitat ist nur verständlich, wenn man der allegierten Literatur eine Aussage entnimmt. So kann das »Verstehen« der einzelnen Quelle letztlich die Mobilisierung eines großen Quellenhintergrundes bedingen. Es kann offenbar kein Ideal der Quellenuntersuchung sein, die einzelne Quelle möglichst zusammenhanglos nur an und für sich auszuwerten.

[11] Es ist möglich und sinnvoll, Rechtsvorstellungen verschiedener historischer Akteure, soweit je für sich quellenmäßig nachvollziehbar, einander gegenüberzustellen, vor allem, sofern diese Akteure als Mitglieder in etwa desselben wissenssoziologischen Kollektivs angesehen werden können. Dies setzt eine wenigstens annähernde Zeitgenossenschaft voraus. Wenn Ulpian und Julian denselben Fall verschieden gelöst haben, ist dies ein fachgeschichtlich $\mathrm{zu}$ erhebender und $\mathrm{zu}$ diskutierender Befund. Ebenso ist es auch ein wissenschaftlich zu behandelnder Befund, wenn man feststellen kann, dass Dokumente aus der Verkehrs- oder der Gerichtspraxis mit Aussagen »theoretischer « Natur übereinstimmen oder nicht übereinstimmen.
[12] Wie es einem Mathematikhistoriker nicht verwehrt ist, in einer Beweisführung Leibniz' einen Gedankensprung zu konstatieren, so ist auch der Rechtshistoriker nicht gehindert, die Stimmigkeit und Schlüssigkeit juristischer Überlegungen der Vergangenheit - vor deren eigenem Zeithintergrund und unter Berücksichtigung der zeitgenössischen Methodik - zu evaluieren. Im Prozess des Verstehens ist es geradezu unvermeidlich, dass man im Quellenmaterial auf Zirkelschlüsse, Selbstwidersprüche, Fehlzitationen oder dergleichen trifft; diese muss der Rechtshistoriker nicht ignorieren in der Sorge, er würde ansonsten nicht mehr historisch arbeiten. Daher ist etwa die Aussage, ein bestimmtes Todesurteil sei ein "Justizmord " gewesen, eine Aussage auf dem Boden der Rechtsgeschichtsforschung, weil und insofern man sinnvoll darüber diskutieren kann, ob das Gericht sich über seinerzeit für verbindlich gehaltene Verfahrensnormen (welche quellenmäßig rekonstruierbar sein müssen) hinweggesetzt hat. Es wäre - wahrscheinlich auch aus der Sicht der Allgemeingeschichte eine Verarmung, wenn man in einer falsch verstandenen Beschränkung auf die Quelle in ihrer materiellen Abgeschlossenheit auf das juristische »Verstehen« verzichten wollte, das die Feststellung von Übereinstimmung mit oder Differenz zu anderen Quelleninhalten einschließt.

[13] Wenn das geschichtswissenschaftliche Erfassen des juristischen Sachgehalts einer Quelle also den Befund eines juristischen »Fehlers« - gemessen am vorherrschenden juristischen Gedankengut der Zeit, soweit dieses rekonstruierbar ist einschließen kann, dann doch nur insoweit, als es sich noch um das Verstehen des Quellenbefundes handelt. Ein »Weiterdenken« des Quellenbefundes mit Mitteln der (seinerzeitigen oder gar der heutigen) juristischen Logik ist nicht mehr Sache quellenbezogener Rechtsgeschichtsforschung. Insofern markiert »Verstehen« zugleich die Grenze des wissenschaftlichen Umgangs mit dem Quellenbestand.

[14] Die vergangene Rechtsordnung "als Ganze«, so wie wir sie für die Gegenwart als Ideal postulieren, ist kein Erkenntnisgegenstand der Rechtsgeschichtsforschung. Es ist nicht möglich, z. B. »Das römische Privatrecht des 2. Jahrhunderts« darzustellen, wenn dabei der Eindruck erweckt würde, man könne hiermit eine durch Lückenlosigkeit ausgezeichnete gedankliche Ordnung beschreiben. Bleibt man bei der geschichtswissenschaftlichen Methode, lässt sich allenfalls 
eine Darstellung dessen geben, was wir bruchstückhaft über einzelne Rechtsaktivitäten und -vorstellungen in Erfahrung bringen könnten. Besonnene Fachvertreter haben derartige Titel auch immer so verstanden, als seien sie etwa wie folgt zu lesen: "Was wir aufgrund unserer Quellen über >Das römische Privatrecht des 2. Jahrhunderts` aussagen können.«Es ist das Ziel der rechtsgeschichtlichen Forschung, dasjenige auszusagen, was unsere Quellen über das Rechtsdenken vergangener Zeiten erkennen lassen. Verkürzt: Rechtsgeschichte erschöptt sich in der Erschließung (Auffindung und Verfügbarmachung) rechtlich relevanter Quellen und in deren juristischem Verstehen.

[15] Es ist unvermeidlich, dass bei der Zusammenstellung von mehreren rechtshistorischen Erkenntnissen mit Bezug zum Rechtsgeschehen einer bestimmten Zeit diese in eine gewisse Reihenfolge gebracht werden. Die Methoden der rechtsgeschichtlichen Forschung erlauben es aber nicht, dem Recht einer vergangenen Zeit eine retrospektive Ordnung zu geben, für die man - gerade in ihrer konkreten Ordnungsstruktur - den Anspruch erheben könnte, sie sei selbst historisch. Hiervon zu unterscheiden ist die mögliche Verfolgung von konkreten historischen Systematisierungsleistungen (»System des Quintus Mucius Scaevola« o. Ä.), die freilich schon in ihrer Zeit regelmäßig nur ein unvollkommenes Abbild des Rechtsganzen gewesen sein dürften.

[16] Zur »Norm» als Gegenstand der rechtsgeschichtlichen Forschung. Gegenstand der Rechtsgeschichtsforschung können nur solche Normsätze sein, die die konkreten Zeitgenossen tatsächlich - einzeln oder in einem kollektiven Einklang postuliert haben: Die "Norm« als gedachter Sollenssatz hat eine vergangene Existenz überhaupt nur insoweit, als sie für Zeitgenossen zum Gedankenmaterial gehört hat, und sie ist weiterhin ein Gegenstand der Rechtsgeschichtsforschung nur unter der Bedingung, dass die stattgefundene Bewegung in den Gedankenwelten vergangener Personen einen Niederschlag in uns zugänglichen Quellen gefunden hat. Ein häufiger Methodenfehler liegt meines Erachtens in der Hypostasierung einer Norm (»im römischen Recht galt, ...«), wo nur eine Einzelfallentscheidung (z. B. des Juristen Julian) historisch belegt ist. Normtexte wiederum sind mit dem früheren wissenssoziologischen Bestand einer Überzeugung von einem rechtlichen Sollen nicht identisch, womöglich aber ein Indiz für diesen.

[17] Zur »Entwicklung« im Bereich von Rechtsgeschichte. Die Wissenskollektive, die Träger des Phänomens »Recht « sind, entstehen in ihrer Zeit nicht jeweils de novo, sondern sie entwickeln sich im Wege personaler Ergänzung und Erneuerung über Generationen hinweg. Die historischen Vorstellungen von rechtlichen Sollenssätzen werden daher von den jeweiligen Akteuren unter dem Eindruck von schon vorgefundenen, ihnen tradierten Rechtsvorstellungen gebildet. Auf dem Weg über die immer wieder neue, in fortgeschrittenen Verhältnissen schul- oder universitätsbasierte Vermittlung von Rechtsanschauungen ergibt sich so etwas wie eine Entwicklung von Normvorstellungen, die auch einen möglichen Untersuchungsgegenstand historischer Forschung bildet. Dies ist keine »Normgeschichte«, wenn man sich darunter einfach eine Abfolge von Normtexten vorstellt. Die »Norm« ist überhaupt keine Entität, die sich an und für sich selbst entwickeln könnte.

[18] Ein Medium der Verständigung über Rechtsvorstellungen sind Rechtsaufzeichnungen der verschiedensten Art. Vor allem bei entwickelten Verhältnissen schul- oder universitätsbasierter Stoffvermittlung erscheinen Darstellungen des Rechtsganzen oder einzelner Materien speziell für den Zweck der juristischen Lehre. Der Inhalt solcher Schriften (an sich »Theorie«) ist natürlich nicht mit der Totalität des zeitgenössischen Rechtsgeschehens gleichzusetzen, er erheischt aber die besondere Beachtung des Rechtshistorikers, weil er dem juristischen Denken der damit Ausgebildeten die Struktur und Begriffe liefert, so dass seine Kenntnis wesentlich zum historischen Verständnis des zeitgenössischen Rechtshandelns beitragen kann. 